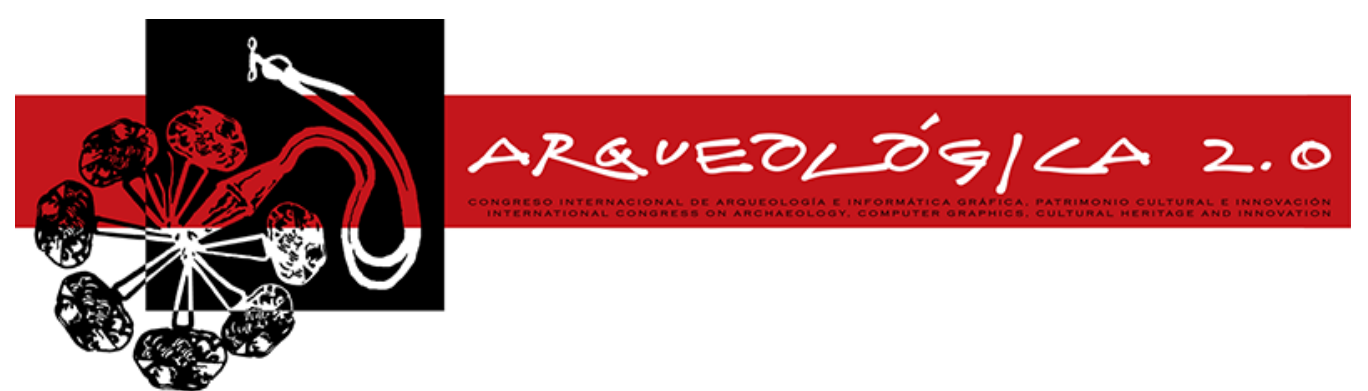

Proceedings of the $8^{\text {th }}$ International Congress

on Archaeology,

Computer Graphics,

Cultural Heritage and Innovation

'ARQUEOLÓGICA 2.0'

in Valencia (Spain),

Sept. $5-7,2016$

DOI: http://dx.doi.org/10.4995/arqueologica8.2016.3517

\title{
METODOLOGÍA Y APLICACION PRÁCTICA PARA LA DIGITALIZACIÓN DE PATRIMONIO COFRADE MEDIANTE LA FUSION DE DATOS DE LÁSER ESCANER Y FOTOGRAMÉTRICOS
}

\author{
METHODOLOGY AND PRACTICAL APPLICATION FOR RELIGIOUS HERITAGE DIGITALIZATION USING \\ SCANNER LASER AND PHOTOGRAMMETRIC DATA
}

\author{
Carlos Colomo*, José Luis Pérez, José Miguel Gómez, Francisco Javier Rosales
}

Dpto. de Ingeniería Cartográfica, Geodésica y Fotogrametría, Escuela Politécnica Superior de Jaén, Campus de las Lagunillas, Universidad de Jaén s/n, 23071 Jaén, España. cmcj0002@red.ujaen.es; ilperez@ujaen.es; iglopez@ujaen.es; fjra0004@red.ujaen.es

\begin{abstract}
:
This paper describes the methodology used to capture information from a religious sculpture. The study also shows the results obtained in using free software and fusion of data from laser scanner and photographs. In this sense, one of the problems that can occur when Heritage documentation is made, is the impossibility of move the object of study from other places with appropriates environment conditions to capture data. This problem is especially important in the case of sculptures found upright with certain height, where many upper portions are hidden by the inability to locate safely locate the instruments. This paper show a case of study with this problem and provides the solution to capture data in cenital position with laser scanner. Finally, the overall results are showed and some considerations in terms of conservation and diffusion of the cultural heritage.
\end{abstract}

Key words: cultural heritage, 3D documentation, instrumentation, data fusion

\section{Resumen:}

En este trabajo se presenta la metodología empleada para la captura de información de una imagen cofrade objeto de estudio, así como los resultados obtenidos en su modelado haciendo uso de software libre y la fusión de datos procedentes de escáner láser y de tomas fotográficas. En este sentido, uno de los problemas que pueden existir a la hora de la documentación tridimensional del patrimonio cultural es la imposibilidad de su desplazamiento a zonas de trabajo donde las condiciones y entorno de trabajo sean los idóneos para llevar a cabo la captura de los datos. Este problema es especialmente importante en el caso de imágenes o esculturas que se encuentran en posición vertical con cierta altura, donde muchas partes superiores se quedan ocultas por la imposibilidad de situar de manera segura el instrumental que suele ser pesado, sobre el objeto a digitalizar. En este trabajo, se presenta un caso de estudio donde aparece esta problemática y la solución aportada para realizar capturas de tipo cenital con láser escáner. Finalmente, se muestran los resultados generales obtenidos, así como algunas consideraciones de los mismos en cuanto a los resultados en cuanto a la conservación como la difusión del objeto modelizado.

Palabras clave: patrimonio cultural, documentación 3D, instrumentación, fusión de datos

\section{Introducción}

El modelado tridimensional se podría describir como la descripción numérica de una superficie a partir de un conjunto de puntos medidos por diferentes técnicas. De esta forma, a partir de datos discretos, se idealiza una superficie matemática a partir de distintos algoritmos de reconstrucción superficial, como son los métodos de Poisson, Ball Pivoting o el VCG (Berger et al. 2014).
En cuanto a las principales técnicas para la obtención de esta información discreta del objeto, podemos diferenciar dos grandes grupos. En un primer lugar, las técnicas fotogramétricas, las cuales basan la obtención de la geometría del objeto de estudio en la reconstrucción de la posición de la fotografías y en la detección de puntos homólogos, de manera que se reconstruye su posición tridimensional a partir de información bidimensional de las imágenes. Por otro lado tenemos los sistemas de escaneado 3D, entre las

"Corresponding Author: Carlos Colomo, cmcj0002@red.ujaen.es 
que destacan dos tipologías diferenciadas; en primer lugar los sistemas de luz estructurada, los cuales basan la reconstrucción geométrica del objeto en la proyección de un patrón de luz sobre el mismo y su registro en un sistema de adquisición. $Y$ por otro lado, los sistemas de escáner de barrido, que proyectan un haz láser que es registrado por el Sistema de visión del escáner. (Sansoni et al. 2009)

Analizando las principales características de los sistemas de captura mediante fotogrametría y sistemas de escáner 3D, así como los resultados obtenidos mediante su uso, se podrían plantear algunas consideraciones:

- Los sistemas de escáner 3D presentan precisiones mayores que las obtenidas por fotogrametría, y obtienen información mediante observación directa sobre el objeto frente a la fotogrametría que la obtiene mediante la interpretación de la información capturada en imágenes.

- El coste de los sistemas de escaneado es mayor que los sistemas necesarios para llevar a cabo estudios fotogramétricos.

- El texturizado mediante fotogrametría tiene una mayor calidad que el que proporcionan algunos sistemas láser.

Por otra parte, sería importante comentar la gran importancia que está teniendo en estos últimos años la irrupción de plataformas de software libre, como MeshLab o Bundler SFM, así como de sistemas de adquisición de datos tridimensionales de bajo coste, aunque es cierto que aunque ofrecen resultados muy visuales, no llegan en ocasiones a conseguir las precisiones y terminaciones obtenidas a partir de otros sistemas comerciales.

\subsection{Técnicas $3 \mathrm{D}$ en el duplicado artístico}

El campo de la reproducción artística, aplicado a la obtención de copias de seguridad, es algo que ha existido desde hace muchos siglos, modificándose los procedimientos utilizados a lo largo del tiempo. Así, ya se tiene constancia del uso del método de la cuadrícula utilizada por los egipcios, evolucionando estas técnicas hasta las ampliadoras, reductoras y máquinas de puntos de finales del siglo pasado. Sin embargo, gracias a la tecnología se ha logrado dar un paso más allá, y a día de hoy es posible la realización de una copia sin intervenir en la imagen para obtener un vaciado, como sí era necesario en las técnicas anteriormente nombradas.

El proceso de obtención de reproducciones exactas o copias de seguridad tridimensionales se considera como la realización de un molde virtual que captura por completo la superficie de la imagen, con una resolución tal que cualquier detalle de la misma queda registrado para su posterior uso o análisis.

El digitalizado tridimensional de imaginería cofrade es una técnica que consiste en la "obtención de modelos digitales" de objetos tridimensionales normalmente a partir del uso de un escáner 3D combinando en algunos casos con imágenes fotográficas. De este modo, y tras el procesado y edición de los datos, se obtienen copias de seguridad en 3D de esculturas, que son un fiel reflejo de la realidad en formato digital, resultado de un meticuloso y laborioso proceso de digitalización 3D con resoluciones inferiores al milímetro.

La implementación de las técnicas digitales para la obtención de copias de seguridad, se debe principalmente a las ventajas que éstas presentan respecto las técnicas de replicado tradicionales de copiado por puntos o usando mascarillas de silicona, entre las que destacan:

- Es inocuo para la talla, ya que en ningún momento existe contacto con la obra de arte.

- Facilidad de custodia, ya que no requiere de un espacio físico como en el caso de las copias tradicionales, por tratarse de un archivo digital.

- Además de no ser necesaria la presencia del objeto en el momento de hacer la copia, dado que una vez digitalizada se podrá hacer la copia en cualquier momento y lugar. Esto es una gran ventaja frente a otros métodos tradicionales donde suele ser necesario que exista el objeto original.

Por otra parte, los resultados obtenidos, aparte de ser muy útiles para la conservación del patrimonio histórico, también constituyen una herramienta de gran potencial para la difusión y puesta en valor del patrimonio que ha sido digitalizado.

\subsection{Objeto de estudio}

La imagen del Santísimo Cristo Yacente (Fig. 1), objeto de estudio, realizada en 1959 por el escultor Constantino Unghuetti, está inspirado en las proporciones del cuerpo de la Sabana Santa de Turín, motivo por el cual mide 1,83 metros. Está realizada en pino de Flandes, policromada en un principio por Francisco Cerezo y en 1961 por Juan Abascal, policromía que ha mantenido hasta su restauración en el año 2005 por Francisco Romero Zafra.

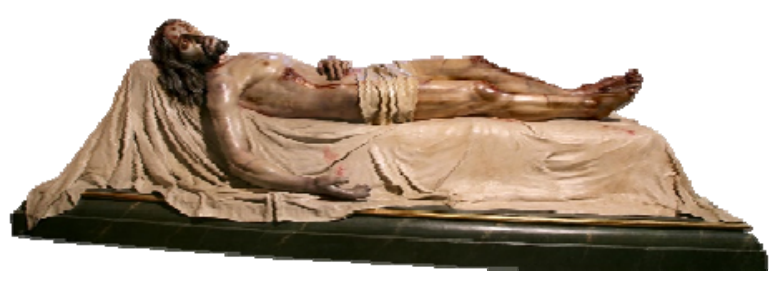

Figura 1: Imagen Objeto de estudio.

\section{Material}

A continuación se presenta el instrumental utilizado para la captura de la información geométrica, así como para la información de la textura del objeto estudiado.

\subsection{Minolta V 910}

Para la realización de este trabajo se ha utilizado el escáner láser Konica Minolta VIVID 910 (Fig. 2). Se trata de un instrumento para digitalización 3D sin contacto, del tipo de triangulación capaz de capturar 300,000 puntos en una única toma. Se trata de un equipo que presenta un volumen variable de escaneo (entre $100 \times 80 \times 40 \mathrm{~mm}$ y $1200 \times 900 \times 400 \mathrm{~mm}$ ) manteniendo la precisión gracias a su sistema de lentes 
intercambiables. Las principales especificaciones técnicas del instrumento se encuentran descritas en la Tabla 1.

Tabla 1: Características Konica Minolta VIVID 910

\begin{tabular}{|c|c|}
\hline Konica Minolta & Características \\
\hline \multirow[t]{3}{*}{ Lentes } & TELE: Distancia Focal f=25mm \\
\hline & MIDDLE: Distancia Focal $\mathrm{f}=14 \mathrm{~mm}$ \\
\hline & WIDE: Distancia Focal $\mathrm{f}=8 \mathrm{~mm}$ \\
\hline Rango de trabajo & Entre 0.6 y $1.2 \mathrm{~m}$ \\
\hline Clase de láser & $\begin{array}{c}\text { Láser de Clase } 2 \text { (IEC 60825-1), "Seguro } \\
\text { para la vista ", Class } 1 \text { (FDA) }\end{array}$ \\
\hline $\begin{array}{l}\text { Método de } \\
\text { barrido del láser }\end{array}$ & Espejo rotatorio \\
\hline Precisión & $X: \pm 0.22 \mathrm{~mm}, Y: \pm 0.16 \mathrm{~mm}, \mathrm{Z}: \pm 0.10 \mathrm{~mm}$ \\
\hline
\end{tabular}

El sistema presenta la posibilidad de capturar imágenes equivalentes a una cámara de 24 bits de color, sin embargo en la práctica, los resultados obtenidos no son los adecuados. Por otro lado, aunque según las especificaciones técnicas el escáner funciona bien en condiciones lumínicas de una oficina en tono a $500 \mathrm{~lx} o$ menos, se ha comprobado que trabaja mejor en condiciones de penumbra u oscuridad, condiciones que afectan negativamente a la captura de la textura. Además, la captura del color con este instrumento presenta muchas irregularidades, ya que se ve muy afectado por las condiciones de luz, el ángulo con el que el haz incide sobre el objeto, variando el tono en función de donde se capture el objeto Por ese motivo, se propone separar la captura del color de la captura de la geometría haciendo uso de cámaras digitales.

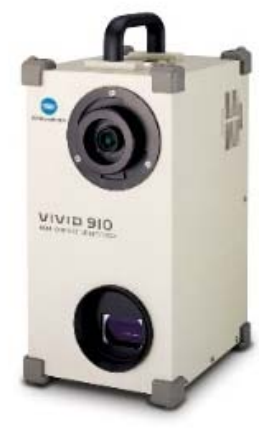

Figura 2: Minolta V910.

\subsection{Cámaras digitales}

Para la captura de la información de la textura del objeto de estudio se han utilizado 2 cámaras digitales, por un lado una cámara compacta Fujifilm finepix F660 EXR y por otro lugar, una cámara tipo bridge Panasonic DMCFZ45, cuyas características aparecen en la Tabla2:

Tabla 2: Características de las cámaras usadas

\begin{tabular}{c|c|c} 
Cámara & Finepix F660 EXR & DMC-FZ45 \\
\hline Tamaño Pixel (mm) & 0.0013 & 0.0014 \\
Focal (mm) & 4.4 & 4.5 \\
Tamaño sensor (pix) & $4608 \times 3456$ & $4320 \times 3240$ \\
Tipo Sensor & CMOS & CCD
\end{tabular}

\section{Proceso de trabajo}

\subsection{Captura de la geometría}

Tal como se ha comentado, el objeto de estudio se trata de una imagen escultórica que representa un cristo yacente. Las principales dificultades encontradas a la hora de realizar la toma de datos son, en primer lugar la distancia existente entre el suelo y la parte más alta de la imagen, y por otro la distancia entre los extremos de la talla y el centro de la misma, al tener la escultura una base de considerable anchura. Estos dos factores, unidos al peso del equipo, que ronda los 11 kilos, y al tipo de trípode disponible, impedían una captura de datos completa de la imagen utilizando únicamente el trípode convencional, apareciendo dos problemas a la hora de realizar la toma. Por un lado la parte más central de la escultura, la correspondiente al esternón, la parte alta de la cabeza o la zona de sombra por la posición de las piernas, no era posible su adquisición debido a la falta de visión por parte del láser al no poder subir lo suficiente el instrumental y darle la inclinación necesaria dentro de unos límites de seguridad para la imagen (Figs. 3 y 4). Por otra parte, además de las zonas que no eran capturadas en la zona del esternón, la zona del pecho, estaba capturada en gran medida con ángulos muy sesgados entre el láser y el objeto de estudio, lo que obligo a la creación de un prototipo de un trípode de captura cenital, con el que se pretendía resolver ambos inconvenientes, descrito en el apartado 3.1.1.

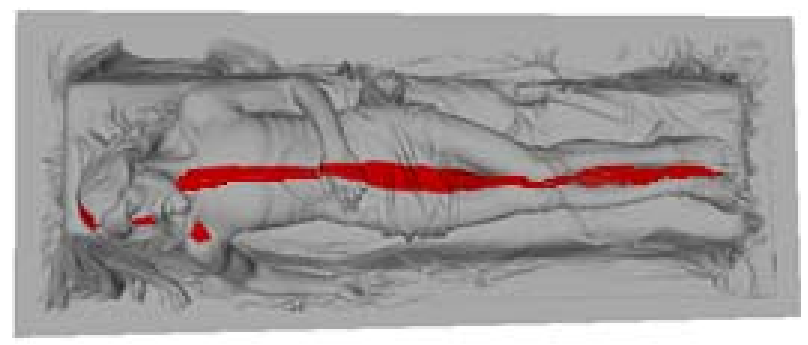

Figura 3: Zonas ocultas con el trípode convencional.

La captura de la información con el escáner ha sido realizada con el objetivo medio, de manera que la planificación para la ejecución del trabajo es de medio metro respecto el objeto. En cuanto a la captura de datos mediante el trípode tradicional alrededor del objeto, se procura que el solape entre las tomas perpendiculares al objeto de estudio sea mínimo del $30 \%$ con los escaneos vecinos para asegurarse un recubrimiento completo y una mayor facilidad para el registro entre escaneos, tanto de manera manual como para un mejor trabajo por parte de los algoritmos automáticos.

Además de la realización de una toma perpendicular hacia el objeto de estudio, desde cada punto de escaneo se realizan dos tomas adicionales con un giro de $45^{\circ}$ a izquierda y derecha, simulando de esta manera tomas convergentes, de manera que se mejora notablemente la captura de zonas de difícil medición con la toma perpendicular minimizando de esta manera zonas ocultas (Fig. 5). Así se tiene una metodología ordenada de trabajo, con una alta redundancia de datos, lo cual facilita en gran medida el trabajo en gabinete y 
reduciendo el riesgo de vuelta al lugar de trabajo por la existencia de zonas sin registrar.

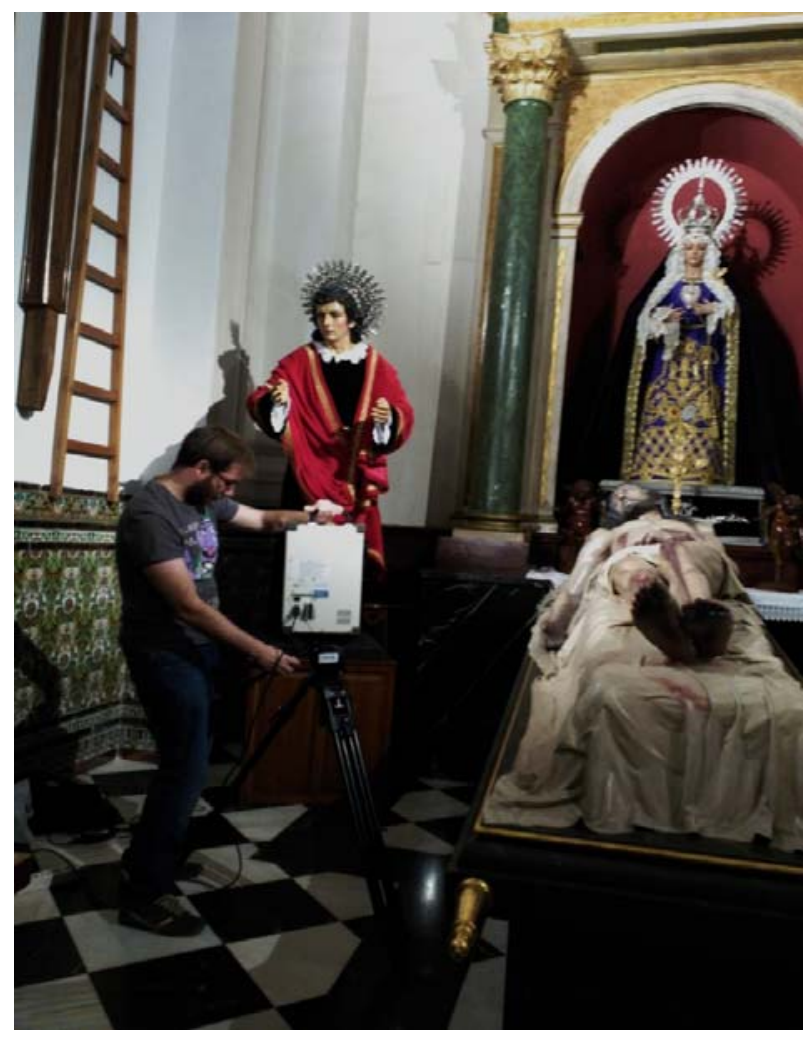

Figura 4: Trabajo Trípode convencional.

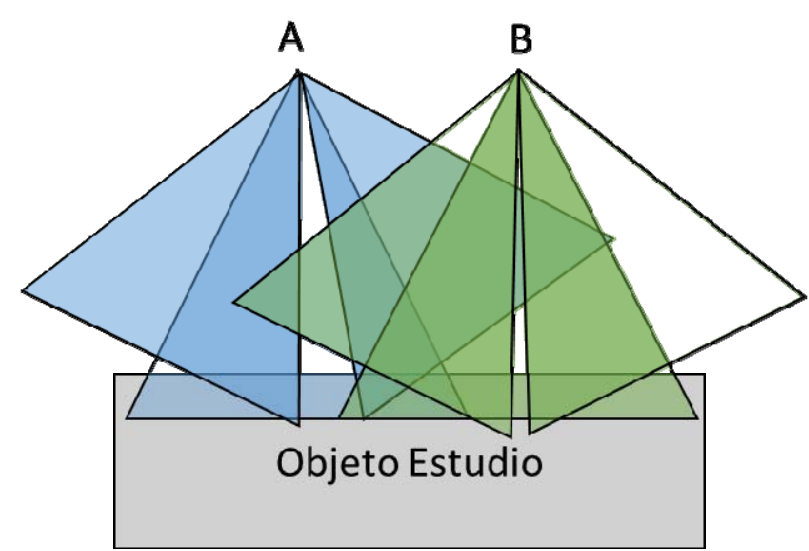

Figura 5: Tomas convergentes con el escáner.

\subsubsection{Prototipo de captura cenital}

La realización de un prototipo para la captura de datos cenitales, debía cubrir la necesidad planteada, y por otro lado deberá ser de fácil montaje y desmontaje, aparte de ser estable para soportar el peso del equipo con total seguridad para la imagen objeto de estudio. Para satisfacer todas estas necesidades se opta por la creación de una estructura dividida a su vez en dos partes funcionales distintas (Fig. 6).

La primera de las partes se trata de una estructura en aluminio de 2 metros de altura, 0.5 metros de largo y 2 metros de ancho que conforma el esqueleto, da rigidez al sistema y sobre la que se apoyará la segunda parte del prototipo. Esta estructura de dos metros se encuentra apoyada en sus cuatro patas por ruedas, dos de ellas equipadas con freno para bloquear el avance de la estructura. Por otra parte, la estructura se encuentra dividida en pasos para la modificación de la altura cada 5 centímetros, de manera que sobre estos pasos se anclará la segunda estructura en función de la altura de trabajo a la que se quiera trabajar. De esta forma queda solucionado el problema del desplazamiento a lo largo del objeto de estudio desde cierta altura en cenital.

La segunda estructura, que se encuentra anclada al esqueleto del prototipo, es aquella que situada a la altura deseada en el armazón nos permitirá desplazar el instrumental transversalmente al objeto de estudio. Para ello, esta estructura de aluminio hace el trabajo de carril sobre el cual se desliza una pieza de conglomerado de 5 centímetros de grosor y con el tamaño del instrumento, con una apertura centrar para la introducción del sistema de medida del escáner. Con este riel se permite el movimiento transversal del escáner a lo ancho del objeto de estudio.

Una vez diseñado, se realiza el montaje y pruebas de carga para comprobar la estabilidad y seguridad que ofrece este nuevo sistema. Para el montaje del prototipo es necesario aproximadamente 10 minutos para el montaje y el chequeo de la correcta colocación de los elementos con una sola persona. Por otra parte, para comprobar la estabilidad y seguridad del sistema se realizó una prueba de carga durante 10 horas, en donde se cargó en la zona diseñada para colocar el escáner un total de 25 kilos, a una altura de 1,75 metros del suelo, dando por superada dicha prueba de carga.

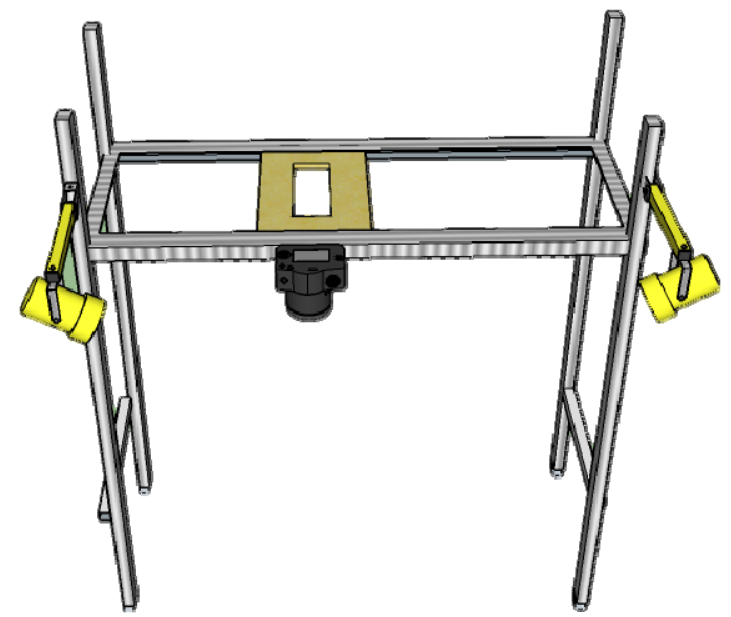

Figura 6: Esquema Prototipo fabricado.

Una vez puesta en funcionamiento en el lugar de trabajo, se comprueba unas más que destacable reducción del tiempo de captura y fatiga del operador al ser los desplazamientos del instrumentar más cómodos para éste, que no tiene que cargar en peso con el escáner y trípode (Fig. 7). Además queda demostrada la eficacia del sistema a la hora de capturar tomas cenitales, ya que además de resolver los problemas que existían a la hora de capturar alguna información, al simular un proyecto de vuelo fotogramétrico, la organización de los datos es óptima para el trabajo posterior de procesado de los mismos (Fig. 8). 


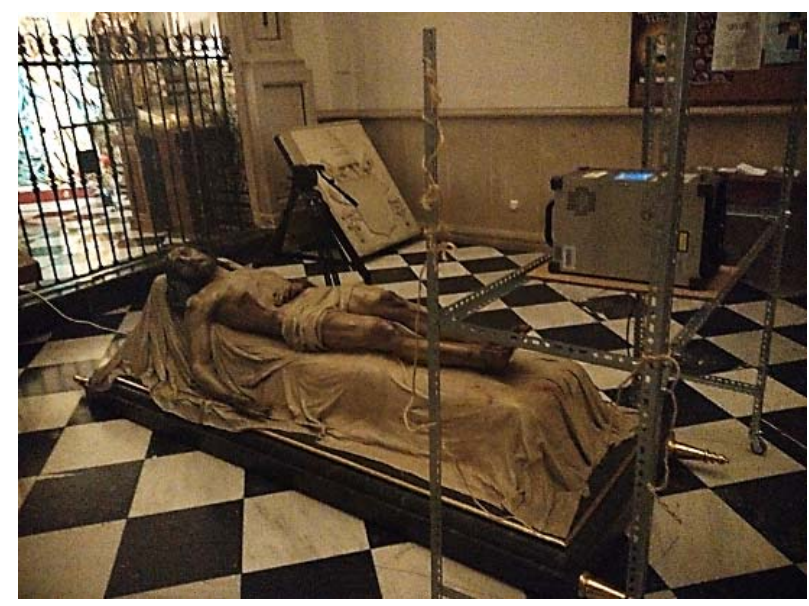

Figura 7: Puesta en funcionamiento prototipo.

Por lo tanto, podemos asegurar que esta estructura permite un mejor flujo de trabajo en este tipo de estudios, al permitir simultáneamente el movimiento longitudinal y transversal a lo largo del objeto mientras se realizan tomas cenitales del mismo muy adecuadas para el estudio de esculturas donde el objeto se encuentre en posición tumbada, pero aplicable también a otro tipos de elementos, como pueden ser mosaicos, los cuales podrían ser estudiados con láser escáner desde una posición cenital sin necesidad de apoyarse sobre el mismo.

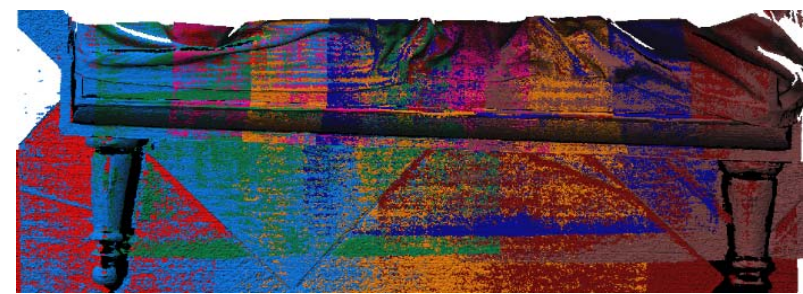

Figura 8: Tomas superpuestas.

Al primer prototipo para captura de tomas cenitales con láser escáner, al comprobar la escasa calidad de las texturas que ofrece el instrumento, se ha realizado una modificación en la cual se ha incorporado a la base de conglomerado un adaptador para situar una cámara fotográfica, la cual se moverá solidaria al escáner, de manera que permita la captura de tomas cenitales, o si se desea la captura de tomas convergentes inclinando la posición de la cámara en función de su posición respecto el objeto.

Este sistema de anclaje de una cámara fotográfica se complementa con la introducción de dos puntos de luz difusa en ambos laterales de la estructura de manera que tengamos totalmente controladas las condiciones lumínicas, mejorando los resultados si realizamos estudios fotogramétricos con software de SfM, los cuales se ven muy afectados con la aparición de reflejos en las imágenes en la realización de los modelos. De esta forma, se podrá trabajar en penumbra, donde el sistema de captura del escáner presenta mejores resultados, al realizar la captura de información geométrica y sin cambiar las condiciones de iluminación ambiental, con este sistema de iluminación difusa obtener las condiciones lumínicas óptimas para la captura fotográfica.

\subsubsection{Reconstrucción de la geometría}

Antes del procesado de los datos del escáner se utiliza primero el software del equipo para transformar el formato nativo .CDM al formato.PLY para llevar a cabo el registro y orientación de las distintas tomas del escáner y modelado de la nube de puntos posterior.

Todos los procesos de alineado manual, ajuste global del alineado, así como el la modelización a partir de la nube de puntos reconstruyendo la superficie ha sido realizado con el software libre MeshLab. De esta manera se pretendía comprobar si era factible con un software libre un flujo de trabajo similar a otros softwares propietarios, con un gran volumen de puntos.

En primer lugar, se han ido relacionando los distintos escaneos a partir de puntos homólogos entre escaneos así con todos los escaneos.

A continuación, una vez pre orientados, se procede a una fase de un nuevo ajuste, de manera que todos los bloques se ajustan simultáneamente minimizando los posibles errores encadenados en los sucesivos alineados de las tomas, ya que al cerrar el objeto tridimensionalmente podemos ajustar el error de cierre.

Una vez la nube completa se ha ajustado, es posible realizar la reconstrucción de la superficie del objeto de estudio, para ello se hizo uso del algoritmo de Poisson, con un total de 18,3 millones de puntos, dando como resultado un modelo de la superficie de la imagen de más de 15,5 millones de polígonos (Figs. 9 y 10).

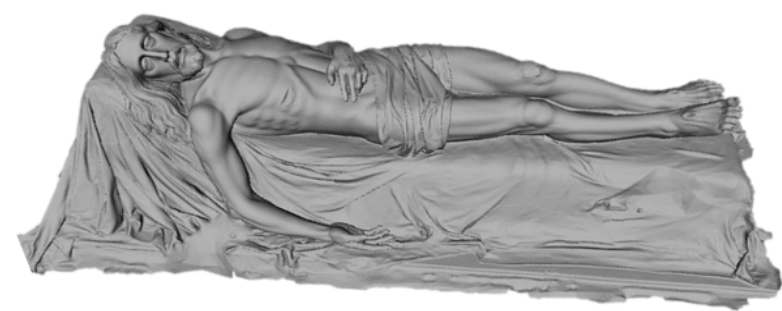

Figura 9: Malla final completa.

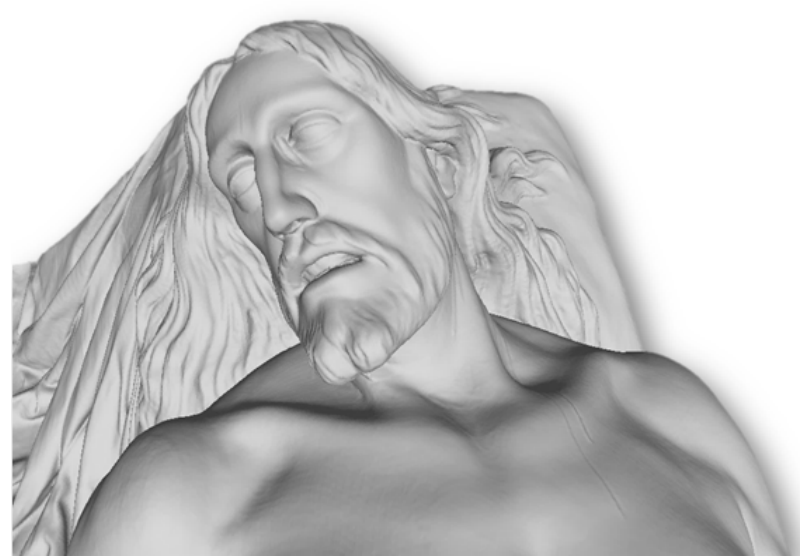

Figura 10: Detalle de la malla, donde se aprecian detalles de la policromía.

\subsection{Captura información de la textura}

En un primer lugar se establece una captura de la información fotográfica únicamente como fuente de estudio complementaria sin pretender realizar modelos 
fotogramétricos, por lo que la geometría de la toma no fue considerada como algo fundamental en el proceso del mismo.

Tal como se ha indicado, la principal función que presentaba el estudio fotográfico era el de servir de guía visual para la relación entre las diversas capturas del láser escáner, el cual puede ser un trabajo complicado si no tenemos información complementaria adicional. Sin embargo,una vez realizado todo el proceso de captura de datos y sin posibilidad de volver a la zona de trabajo, se procede a la obtención de un modelo de la talla mediante fotogrametría, aunque las tomas no habían sido planeadas ni realizadas con suficiente rigor para llevar a cabo un estudio fotogramétrico completo. Debido a esta circunstancia los resultados no son adecuados. El principal inconveniente que aparece es que las imágenes correspondientes a las zonas izquierda y derecha de la escultura no se pueden orientar en un mismo bloque, ya que la zona central de la misma presenta poco solape entre los bloques tomados en cada uno de los extremos. Esto imposibilita en un primer instante la realización de un modelo fotogramétrico completo de la imagen objeto de estudio, de manera que para la obtención de un modelo texturizado completo será necesario la fusión de los datos fotogramétricos y de escáner.

\subsection{Fusión de los datos}

Se parte por un lado de un modelo geométrico de muy alta resolución sin textura, y por otro lado, una fuente importante de información de la policromía que será necesario fusionar para obtener el modelo virtual con textura (Fig. 11).

Con este fin, se realiza la extracción de puntos comunes reconocibles en ambos sistemas y así poder unificar toda la información en un solo sistema, posibilitando la obtención del modelo texturizado. Para la fusión de los datos capturados por el escáner y los datos de imágenes, el proceso llevado a cabo ha sido:

- Extracción de puntos de control en la geometría

- Medición de puntos de control en las imágenes

- Fusión de los bloques independientes

- Combinación del modelo e imagines

- Texturizado

\subsubsection{Extracción de puntos de control modelo}

En primer lugar, para poder combinar dos sistemas de referencia, es fundamental la medición de puntos comunes que hagan de puntos de control de un sistema de referencia a otro. Para ello, el dato que se usa como Sistema de referencia sobre el que trabajar será el modelo triangulado de alta resolución medido con el escáner. Para ello, se han ido extrayendo de manera manual aquellos detalles que podrían ser observables en las imagines fotográficas, como elementos anatómicos de la imagen fácilmente reconocibles como pueden ser heridas, o uñas, pero también han sido medidos elementos de la policromía que han sido capturados por el Sistema de captura geométrica, como son los surcos generados por la sangre (ver Fig. 11).
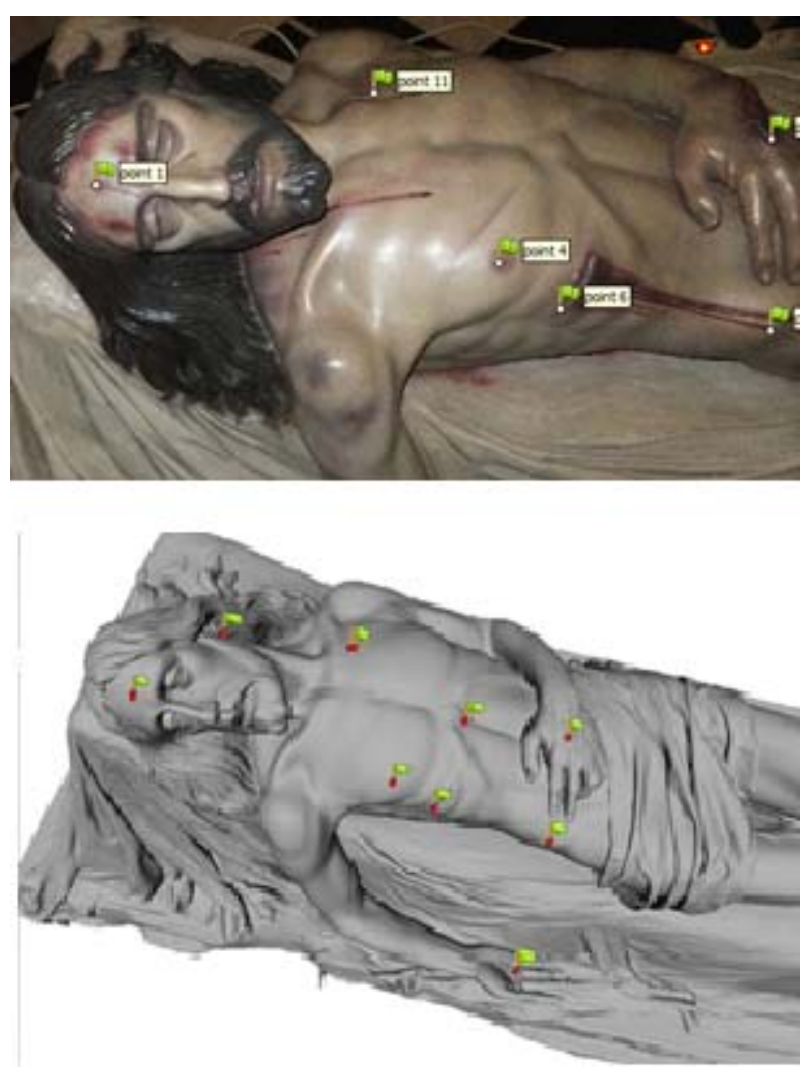

Figura 11: Puntos de control sobre imagen (arriba) y modelo (abajo).

\subsubsection{Medición de puntos de control y fusión de bloques}

Una vez extraídos los puntos de control, se realiza la medición de los mismos en las imágenes correspondientes a los dos bloques fotogramétricos previamente creados. De manera que el problema existente de falta de solape suficiente entre estos dos bloques queda solventado mediante la orientación independiente de cada uno de ellos.

En este punto, se estaría en disposición de poder reconstruir la geometría completa de la talla mediante fotogrametría. Aun así la calidad obtenida realizando el modelado con las imágenes es muy inferior a la obtenida con el láser escáner, por lo que se descarta usar el modelo fotogramétrico.

\subsubsection{Combinación modelo-fotografías}

La filosofía del método es usar la fuente de mayor calidad que tengamos para cada fase del proyecto, por lo que en el caso de la texturización de la malla se utilizará la superficie obtenida a partir del escáner y las imágenes fotogramétricas una vez orientado todo en el mismo sistema de referencia, descartando el uso de la malla obtenida con fotogrametría como base para ser texturizado. De este modo importamos el modelo del escáner en el software fotogramétrico, de manera que también este software nos permite la reducción de la malla en función del uso que se le dará al modelo texturizado (Fig. 12). 


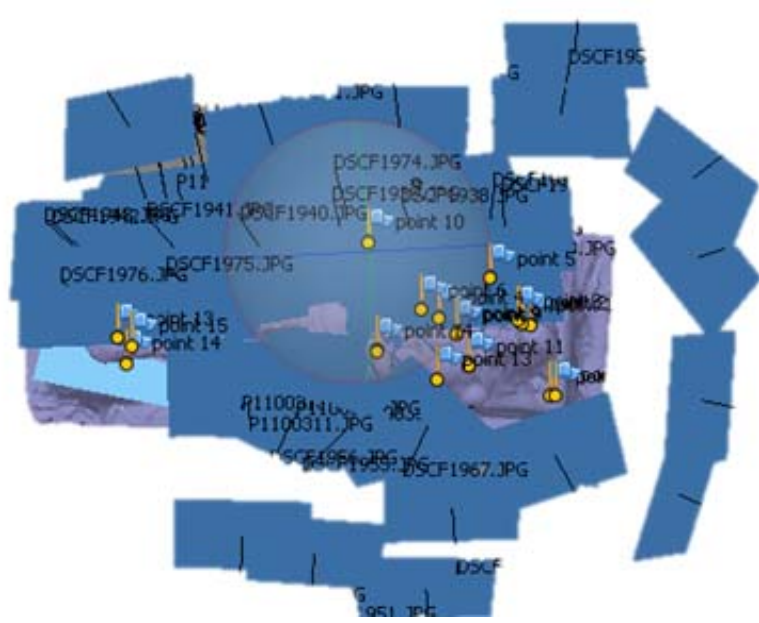

Figura 12: Situación de las imágenes y modelo de alta resolución.

\section{Resultados}

En cuanto a los resultados obtenidos, podemos destacar que con este tipo de técnicas se permite un paso de lo físico a lo virtual mediante las técnicas de adquisición de datos, pero también el proceso contrario, es posible un paso de lo virtual a lo físico en caso de necesidad, con los variados sistemas que existen en el mercado, como son las máquinas de mecanizado o las impresoras 3D. En función de la utilidad con la que se utilicen los datos obtenidos, podemos diferenciar aplicaciones destinadas a la conservación del patrimonio, o aplicaciones de difusión del mismo.

\subsection{Resultados de conservación}

Para los productos de conservación del patrimonio hacienda uso de técnicas geométricas y de imagen, generalmente se hará uso de los productos obtenidos con la máxima precisión en el flujo de trabajo, es decir, los productos brutos ya procesados. En cuanto a las principales aplicaciones que presenta, se podría destacar la realización de copias de seguridad del mismo, de manera que se pueda realizar una reproducción exacta de elementos dañados o de la talla completa en caso de necesidad. Otra aplicación muy recurrente son los estudios multitemporales de manera que se puede estudiar el comportamiento y evolución de los diferentes bloques estructurales que componen estas esculturas, así como multitud de estudios científicos-artísticos de estudio de proporciones.

\subsection{Resultados de difusión}

En cuanto a los resultados destinados a la difusión, se podrían considerar como productos derivados del resultado inicial, de manera que pueden sufrir un proceso de simplificación o transformación a otros resultados. Así los productos derivados para la difusión del patrimonio consisten en reproducciones a diferentes escales y materiales, gracias a las impresoras 3D, publicación en formatos digitales, como son el pdf3D, visores Web, así como aplicaciones de realidad aumentada, virtualizaciones o vídeos de promoción (Figs. 13 y 14).

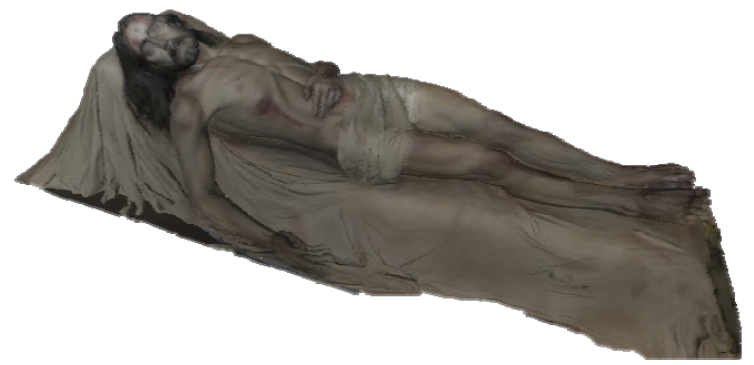

Figura 13: Modelo Texturizado para visor WEB.

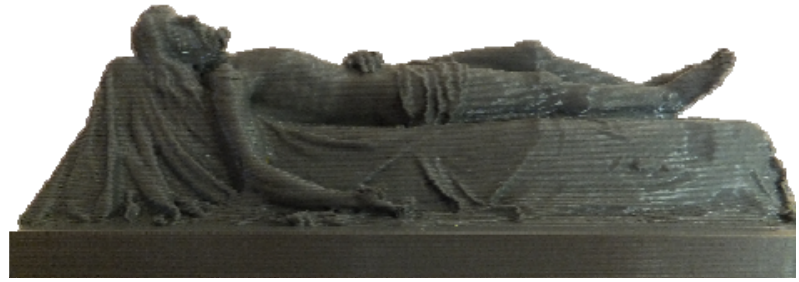

Figura 14: Impresión 3D.

Como conclusiones finales, destacar los resultados satisfactorios obtenidos en cada una de las fases comentadas, de manera que se han obtenidos productos de muy alta resolución geométrica y radiométrica haciendo uso de la fusión de datos. Además el uso del prototipo ha cumplido las expectativas, al agilizar el flujo de trabajo $y$ disminuyendo la fatiga y dificultades del operador y por último recalcar que la posibilidad que permite este tipo de técnicas a la hora de la realización de reproducciones exactas del patrimonio analizado, sin la necesidad de que la imagen sufra contacto alguno ni necesidad de desplazamiento de su lugar habitual.

\section{Agradecimientos}

Agradecimientos al departamento de Ingeniería Cartográfica, Geodésica y Fotogrametría y al Grupo de Investigación Sistemas Fotogramétricos y Topométricos, por facilitar el material para la realización del presente trabajo, así como por el uso de la impresora 3D.

Agradecer también a la Pontificia y Real Cofradía del Stmo. Cristo Yacente y Siervos de Ntra. Sra. De la Soledad por permitir la difusión de los resultados finales.

\section{Referencias}

BERger, M., TAGLIASACCHI, A., SEVERSKY, L., ALLIEZ, P., LEVINE, J., SHARF, A. y SILVA, C., 2014. State of the art in surface reconstruction from point clouds. In EUROGRAPHICS star reports Vol. 1, No. 1, pp. 161-185. DOI:10.2312/egst.20141040

LEVOY, M., PULLI, K., CURLESS, B., RUSINKIEWICZ, S., KOLLER, D., PEREIRA, L. y SHADE, J., 2000. The digital Michelangelo project: 3D scanning of large statues. In Proceedings of the 27th annual conference on Computer 
graphics and interactive techniques, pp. 131-144. ACM Press/Addison-Wesley Publishing Co. doi:10.1145/344779.344849

LOPEZ-MENCHERO, V.M., GRANDE, A., 2011. The principles of the Seville charter. In CIPA symposium proceedings Vol. 2011, pp. 2-6.

SANSONI, G., TREBESCHI, M. y DOCCHIO, F., 2009. "State-Of-The-Art and Applications of 3D Imaging Sensors in Industry, Cultural Heritage, Medicine, and Criminal Investigation" en Sensors, $n^{\circ}$ 9. Multidisciplinary Digital Publishing Institute. Págs: 568-601. DOI:10.3390/s90100568 